\title{
Reorganization energy of electron transfer in viscous
}

\section{solvents above the glass transition: Supporting Information}

\author{
Pradip K. Ghorai and Dmitry V. Matyushov* \\ Department of Chemistry and Biochemistry and the Center for the Early Events in Photosynthesis, \\ Arizona State University, PO Box 871604, Tempe, AZ 85287-1604
}

Atomic charges of $p$-nitroaniline were obtained by fitting the electrostatic potential from $a b$ initio electron structure calculations using GAUSSIAN'03. The ground state geometry of $p$ nitroaniline was obtained on the MP2 level $\left(6-31+\mathrm{G}^{*}\right)$ using X-ray data for the initial geometry. The excited-state charge distribution is obtained from SCI calculations. The results of electronic structure calculations are listed in Table I

TABLE I: Atomic coordinates, ground and excited charges of $p$-nitroaniline.

\begin{tabular}{|c|c|c|c|c|c|}
\hline atom & $\mathrm{x}(\AA)$ & $\mathrm{y}(\AA)$ & $\mathrm{z}(\AA)$ & gs charge $(\mathrm{e})$ & es charge $(\mathrm{e})$ \\
\hline $\mathrm{O}$ & 5.201991 & 3.692969 & 7.447498 & -0.432486 & -0.478180 \\
$\mathrm{O}$ & 5.420828 & 5.489859 & 6.190092 & -0.430114 & -0.486904 \\
$\mathrm{~N}\left(\mathrm{NH}_{2}\right)$ & 10.160594 & 1.971182 & 3.917766 & -0.781180 & -0.699305 \\
$\mathrm{~N}^{\left(\mathrm{NO}_{2}\right)}$ & 5.764136 & 4.346843 & 6.548080 & 0.543659 & 0.414532 \\
$\mathrm{C}$ & 9.038790 & 2.533609 & 4.530267 & 0.198336 & 0.101662 \\
$\mathrm{C}$ & 8.411448 & 1.882589 & 5.607069 & -0.052943 & 0.099222 \\
$\mathrm{C}$ & 7.327806 & 2.461856 & 6.260981 & -0.459211 & -0.621246 \\
$\mathrm{C}$ & 6.908694 & 3.733919 & 5.871161 & 0.432073 & 0.737503 \\
$\mathrm{C}$ & 7.514418 & 4.410958 & 4.812671 & -0.459367 & -0.618722 \\
$\mathrm{C}$ & 8.596379 & 3.818169 & 4.168174 & -0.051696 & 0.098223 \\
$\mathrm{H}$ & 8.752819 & 0.894826 & 5.912630 & 0.145917 & 0.133574 \\
$\mathrm{H}$ & 6.834008 & 1.954221 & 7.083371 & 0.251730 & 0.248066 \\
$\mathrm{H}$ & 7.164255 & 5.397370 & 4.525762 & 0.250029 & 0.246552 \\
$\mathrm{H}$ & 9.081726 & 4.343778 & 3.347340 & 0.146019 & 0.133822 \\
$\mathrm{H}\left(\mathrm{NH}_{2}\right)$ & 10.219709 & 0.958982 & 3.974255 & 0.349884 & 0.345891 \\
$\mathrm{H}\left(\mathrm{NH}_{2}\right)$ & 10.346197 & 2.299372 & 2.974903 & 0.349349 & 0.345309 \\
\hline
\end{tabular}

The Stokes shift correlation function $S(t)$ was fitted to a biphasic form containing the Gaussian (G) and stretching exponential (E) parts

$$
S(t)=A_{G} e^{-\left(t / \tau_{G}\right)^{2}}+\left(1-A_{G}\right) e^{-\left(t / \tau_{E}\right)^{\beta}} .
$$

${ }^{*}$ E-mail:dmitrym@asu.edu . 
TABLE II: The values of the fitting parameters in eq 1.

\begin{tabular}{|c|c|c|c|c|}
\hline $\mathrm{T}(\mathrm{K})$ & $\mathrm{A}_{G}$ & $\tau_{G}(\mathrm{ps})$ & $\tau_{E}(\mathrm{ps})$ & $\beta$ \\
\hline 509 & 0.50 & 0.035 & 0.111 & 1.0 \\
478 & 0.43 & 0.035 & 0.112 & 1.0 \\
448 & 0.47 & 0.035 & 0.128 & 1.0 \\
415 & 0.43 & 0.042 & 0.140 & 1.0 \\
377 & 0.50 & 0.062 & 0.181 & 1.0 \\
359 & 0.46 & 0.057 & 0.217 & 1.0 \\
344 & 0.53 & 0.064 & 0.257 & 1.0 \\
333 & 0.51 & 0.067 & 0.243 & 1.0 \\
316 & 0.57 & 0.073 & 0.362 & 1.0 \\
290 & 0.66 & 0.083 & 0.754 & 1.0 \\
272 & 0.54 & 0.080 & 1.056 & 0.67 \\
249 & 0.54 & 0.096 & 5.973 & 0.44 \\
240 & 0.54 & 0.100 & 11.460 & 0.34 \\
219 & 0.53 & 0.096 & 38.461 & 0.34 \\
\hline
\end{tabular}

The amplitude $A_{G}$ and the Gaussian, $\tau_{G}$, and exponential, $\tau_{E}$, relaxation times as functions of temperature are listed in Table II.

In Figues 1 and 2 we show the results of mean squared diesplacement calculations in SPC/E water. Figure 3 compares the one-particle relaxation time $\tau_{1}(T)$ obtained from analyzing eq 2 with previously reported (ref 16) results by Sciortino et al..

$$
C_{1}(t)=\langle\mathbf{m}(t) \cdot \mathbf{m}(0)\rangle /\left\langle\mathbf{m}(0)^{2}\right\rangle
$$
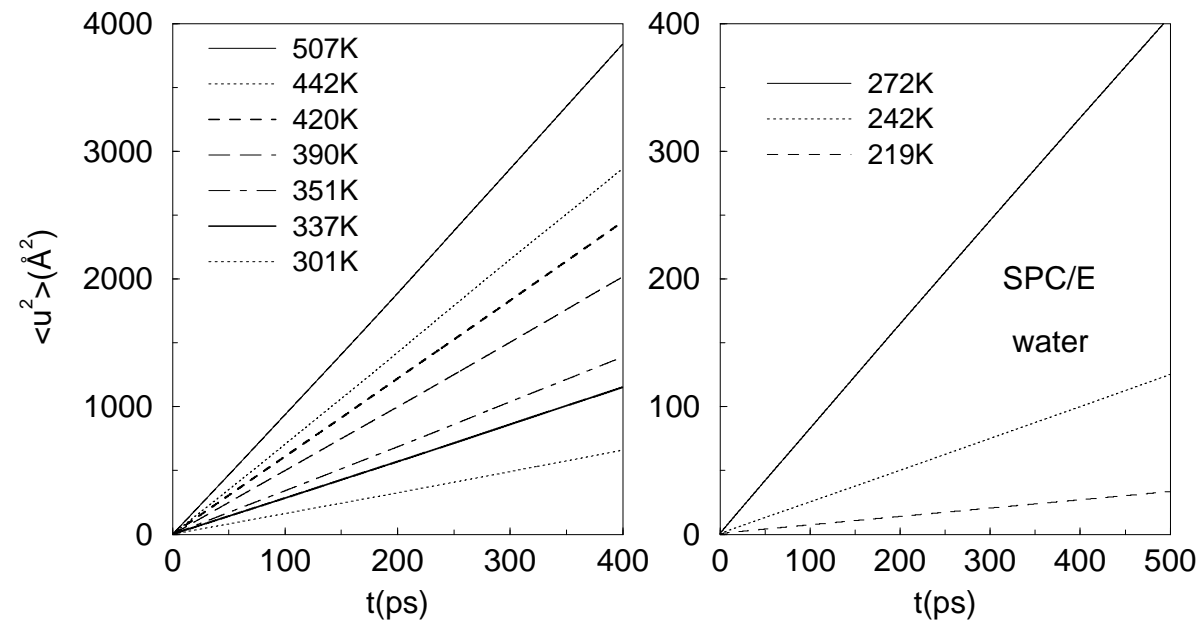

FIG. 1: Mean squared displacement (m.s.d.) of SPC/E water at various temperatures obtained from NVE MD simulations. 


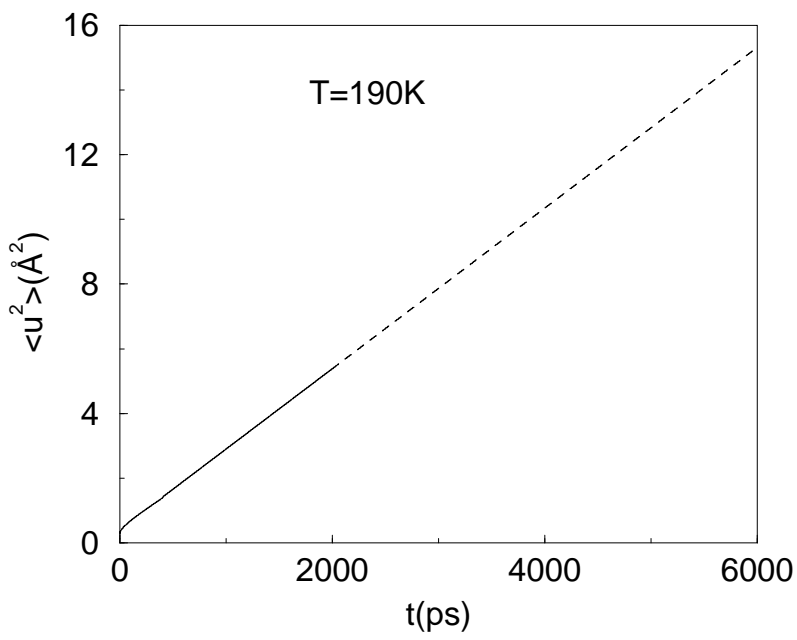

FIG. 2: Mean squared displacement(m.s.d.) of water at 190K. The solid line is from simulations (2 ns), the dashed line shows the extrapolation up to 6 ns of simulation time.

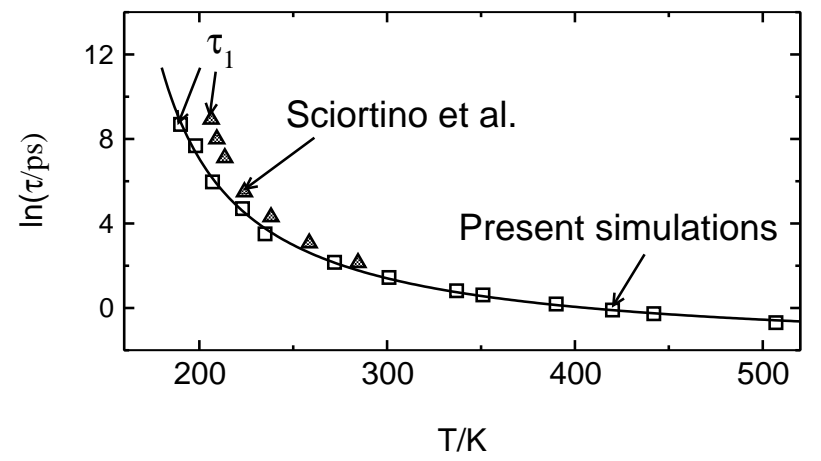

FIG. 3: Correletion time $\tau_{1}(T)$ from Sciortino et al. (ref 16) and from present simulations. 